\title{
Fluorescent imaging sheds new light on apoptosis
}

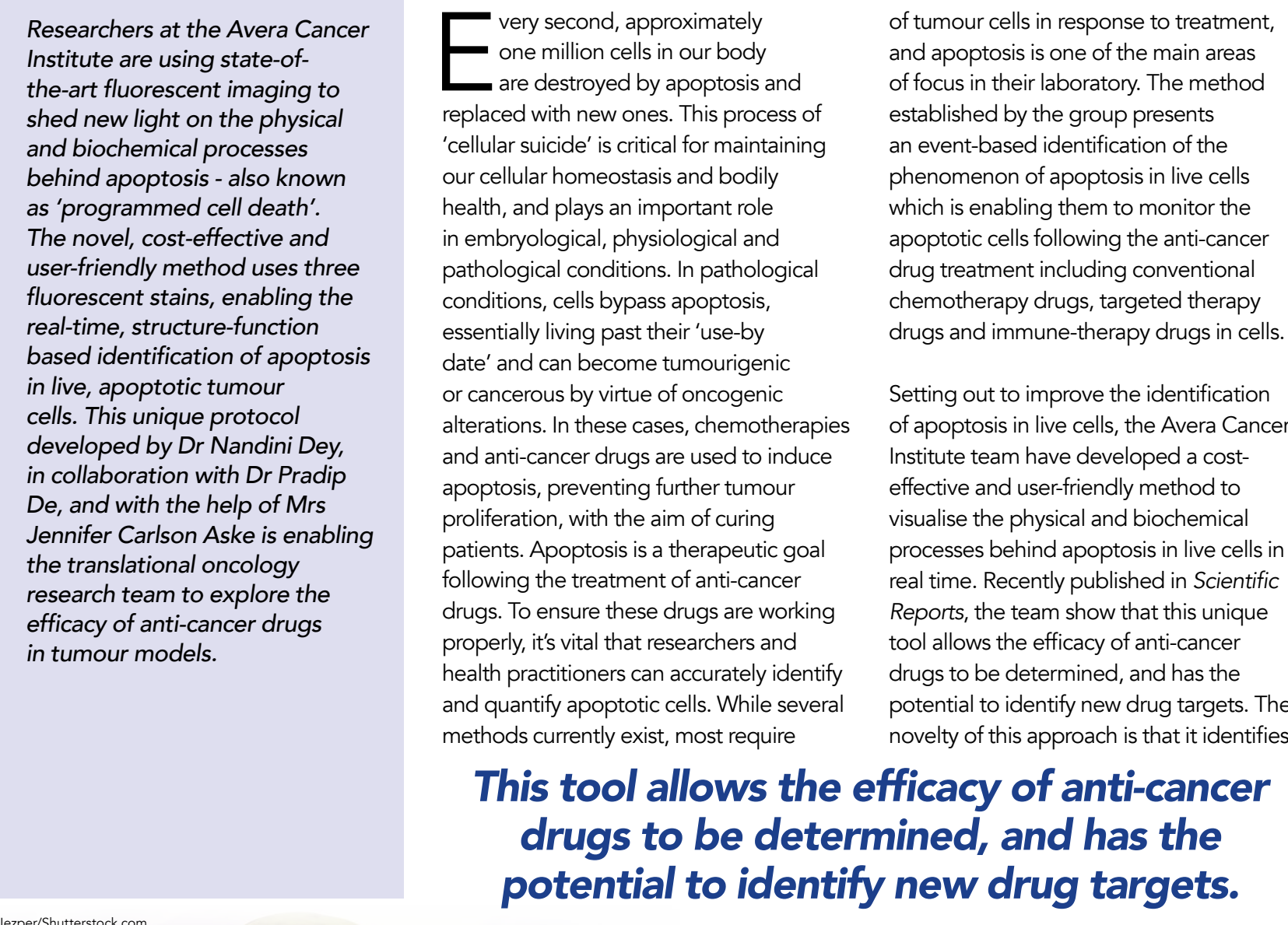

of tumour cells in response to treatment, and apoptosis is one of the main areas of focus in their laboratory. The method established by the group presents an event-based identification of the phenomenon of apoptosis in live cells which is enabling them to monitor the apoptotic cells following the anti-cancer drug treatment inclualing conventional drugs and immune-therapy drugs in cells. Setting out to improve the identification Institute team have developed a costeffective and user-friendly method to visualise the physical and biochemical processes behind apoptosis in live cells in real time. Recently published in Scientific Reports, the team show that this unique tool allows the efficacy of anti-cancer drugs to be determined, and has the potential to identify new drug targets. The methods currently exist, most require This tool allows the efficacy of anti-cancer drugs to be determined, and has the potential to identify new drug targets.

expensive and sophisticated equipment incredibly high resolution microscopy or specialised confocal microscopy. In addition, these methods use processed cell samples, rather than live cultures and cannot show the drugs' mechanisms of (tion in the process of inducing apoptosis in a standard laboratory set-up.

Changing this are Dr Nandini Dey Dr Pradip De and Ms Jennifer Carlso Aske, based at the Avera Cancer studying anti-cancer drugs and their mode of action. In particular, their interest lies in the behaviour three sequential cardinal biochemical, of apoptosis in a laboratory-friendly way.

\section{WHAT IS APOPTOSIS?} In a time of crisis, would you sacrifice do: they are genetically programmed to 'self-destruct' if they suffer irreparable DNA damage, protecting the body from further harm. This 'programmed cell death is called apoptosis. Apoptosis is the hallmark of cancers. It involves certain morphological, biochemical, and physiological sequential steps controlled by a dying cell. Apoptosis shrinkage and loss of nuclear membran is characterised by physical signs
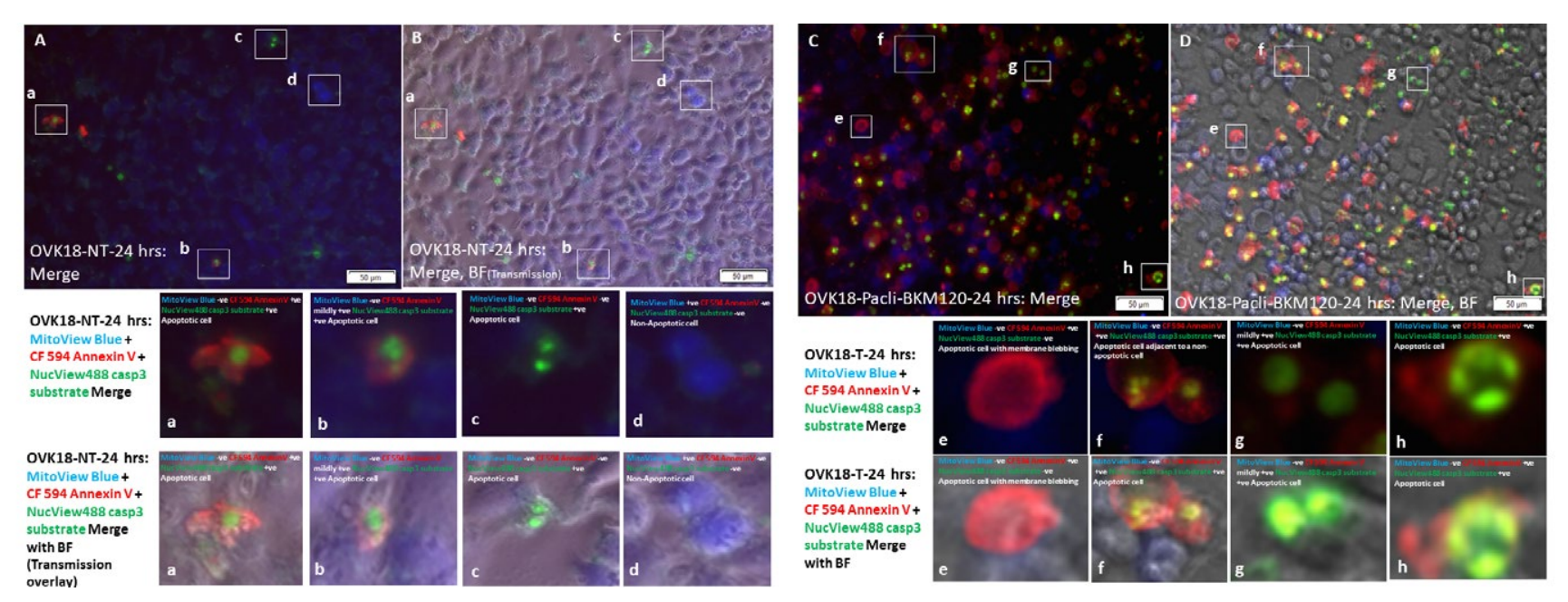

Live triple-fluorescence in OVK18 cells treated with paclitaxel plus BKM120. Live OVK 18 cells were stained with Mitoview Blue + NucView 488 Casp 3
substrate +CF 594 Annexin V (A-D). Insets represent non-apoptotic and eachtotic cells with various combination of staining as mentioned within
each inset (a-d for non-treated cells and e-h for treated cells). In separate
experiments, the validation of the effect of pactititaxel (2.5nM) plus BKMMI20 $\mu M$ ) was tested by the changes in real-time proliferation (E), mitochondria
potential $(E)$-exd apoptosis by fllow cyytometry (H).

integrity, plasma membrane blebbing, nuclear fragmentation and finally,

cell fragmentation.

Apoptotic cells also undergo a series of biochemical changes including executioner caspase activation, mitochondrial membrane alterations and release of a protein called cytochrome C, externalisation of phosphatidylserine - an 'eat me' signal for scavenging macrophages - to the cell surface, poly (ADP-ribose) polymerase (PARP) cleavage nd nuclear DNA fragmentation.

By tracking these well-regulated and equential processes, researchers can cancer drug treatments on tumour cells and potentially identify new drug targets. This is where the research of $\mathrm{Dr}$ Dey and her group comes in.

APOPTOSIS IN A NEW LIGHT Using a fluorescence microscope and three fluorescent dyes (fluorophores), the Avera Cancer Institute team have coupled these three sequentially occurring rate-limiting features of apoptosis in live cells. In their 'triple-fluorescence staining', they can observe the enzymatic activity of executioner caspase 3 green chosphatidy extemal presentation of phosphardyserne (red hionophore) and altered mitochondrial functions (blye

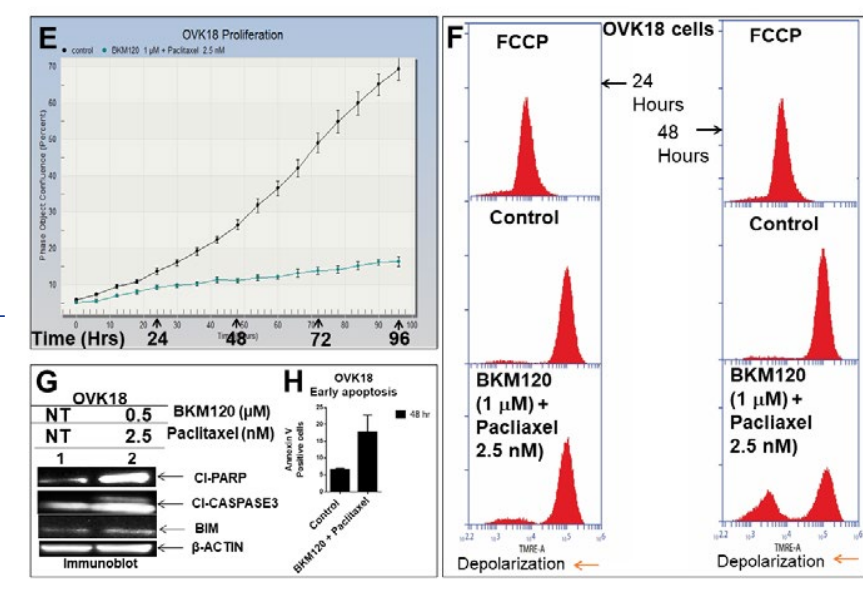
apoptosis, the team treated two ovarian cancer and A2780 with anti-cancer

drugs called paclitaxel (conventional chemotherapy drug) and BMK120 (targeted pan PI3K inhibitor), either alone or in combination. The cells' fluorescent pattenswere then compared with those of untreated cells.

Upon examination under the microscope the researchers found that most untreated To ensure that the anti-cancer drugs were

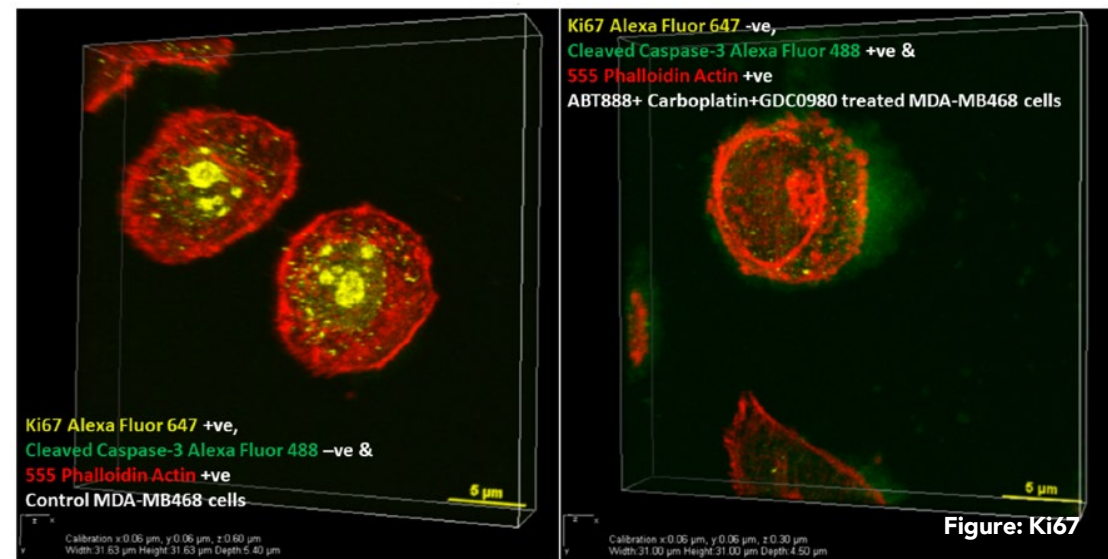

K 167 and cleaved Caspase3 3 double staining of fixed MDA-MB468 cells, control and treated (ABT888-

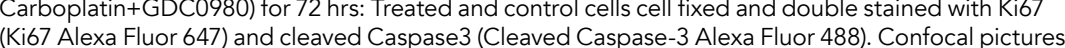

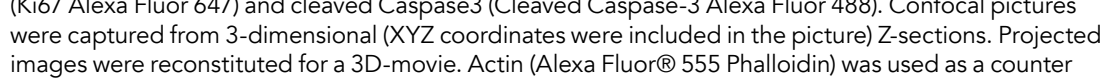

cells fluoresced bright blue, since their mitochondiria were still intact. In contrast, This was 3 initition acted given that caspase presentation only occur during apoptosis. VAUDATING THE CONCEPT 
responsible for these apoptotic effects, cancer cells with different doses of paclitaxel or paclitaxel and BMK120 and pacliaxel or pacltaxel and $B M K 120$ and and at different time points.

Unsurprisingly, proliferation of paclitaxetreated OVK18 cells decreased or stopped, depending on the dose given, with apoptosis setting in after 48 hours and the simultaneous expression of apoptotic markers. Furthermore, these samples had a larger number of dead cells overall, compared to the untreated samples. Similar results were seen in paclitaxel and BMK120-treated OVK18 cells, with apoptosis setting in at 48 hours, however, this time, changes in ppeared at 24 hours.

Paclitaxel-treated A2780 cells took the longest to reach an apoptotic state, with the process occurring 48 and 72 hours after treatment. However, changes in mitochondrial activity occurred 24 and 48 hours post-treatment. This sample also had a larger number of dead cells than the untreated samples, proving that the paclitaxel causes cell death, therefore verifying the microscopy results. Thus using this protocol the group successfully identified the temporal and eventual pattern of drugaction in inducing apoptosis.

\section{IDENTIFYING LIVE}

AND DEAD CELLS

Dorder to identify living and dead cells, samples with a new set of fluorophor

Dead cells fluoresced red while live cells were green. The mitochondial potentia was measured by flow cytometer while live-dead cells were read under fluorescent microscope. Fluorescent

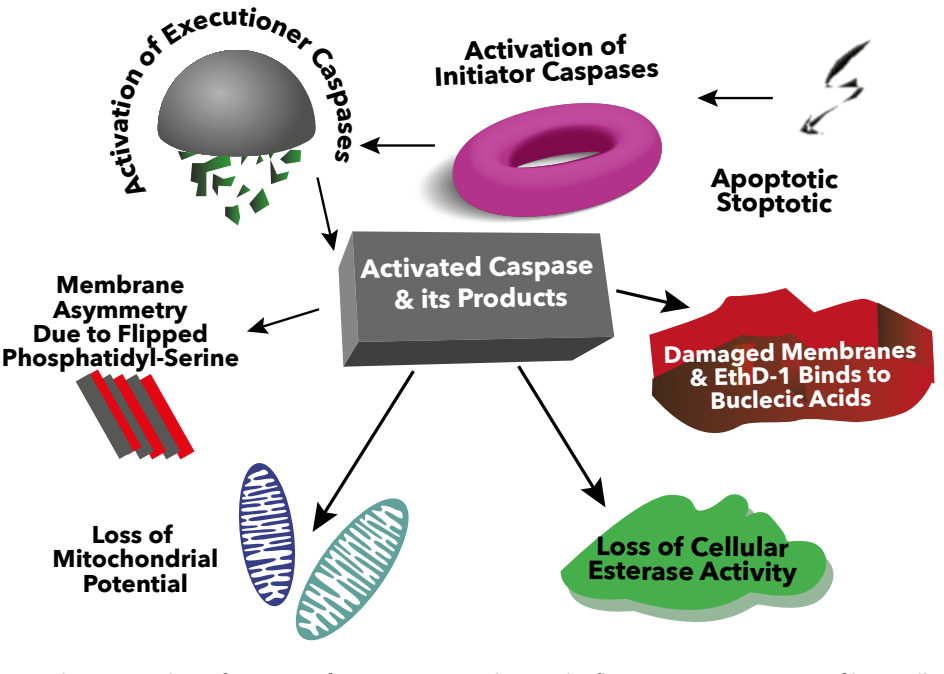

Every second, approx one million cells in our body are destroyed by apoptosis and replaced with new ones.

imaging comparisons using the 'live and still displayed mitochondrial activity dead cell assay' showed no overlapping (blue fluorescence). green (live) colls

In contrast, fluorescent imaging comparisons using their 'triple-
fluorescence staining assay' showed overlapping of red (Annexin V positive apoptotic cells) and green (active caspase 3 positive apoptotic cells) in treated and untreated samples; this is because green cells and red cells were at different stages of apoptosis. No overlap was observed between is to and red and/or green cells, and is to be expected since blue cells with to exhibit apoptosis, while red and or green or both red and green cells were less likely to exhibit functionat blue mitochondria Interestingly. however, a few drug treated cells

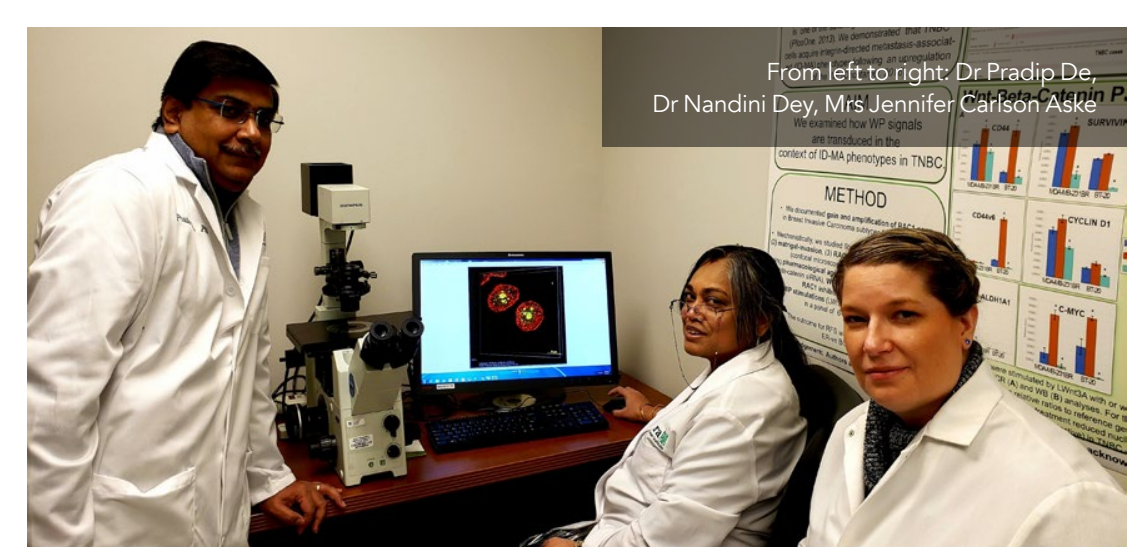

WHY IS THIS IMPORTANT? While not all of these biochemical and apoptotic events are stage-, timeand stimuli-dependent, the strength of the triple-fluorescence staining method lies in its ease of use and its ability to simultaneously stain three apoptotic steps - making it much easier to accurately identify apoptotic versus non-apoptotic cells. In comparison, apoptotic (but not necessarily apoptosisand quantify these cells. Further triple-fluorescence staining can show apoptotic processes in live cells in real time - allowing researchers to see anticancer drugs' mechanisms of action, and their efficacy.

This laboratory friendly method can be standardized with minimalistic laborato equipment and resources, making it a relatively cheap and accessible method that can be applied to any relevant drug treatment. Notably, it has already been used to observe the effects of different drug combinations on other tumour cell lines. The strength of this assay lies in its ability to identify modes of action of pro-apoptotic dr
based manner. critical morphological and biochemical most existing techniques rely on one

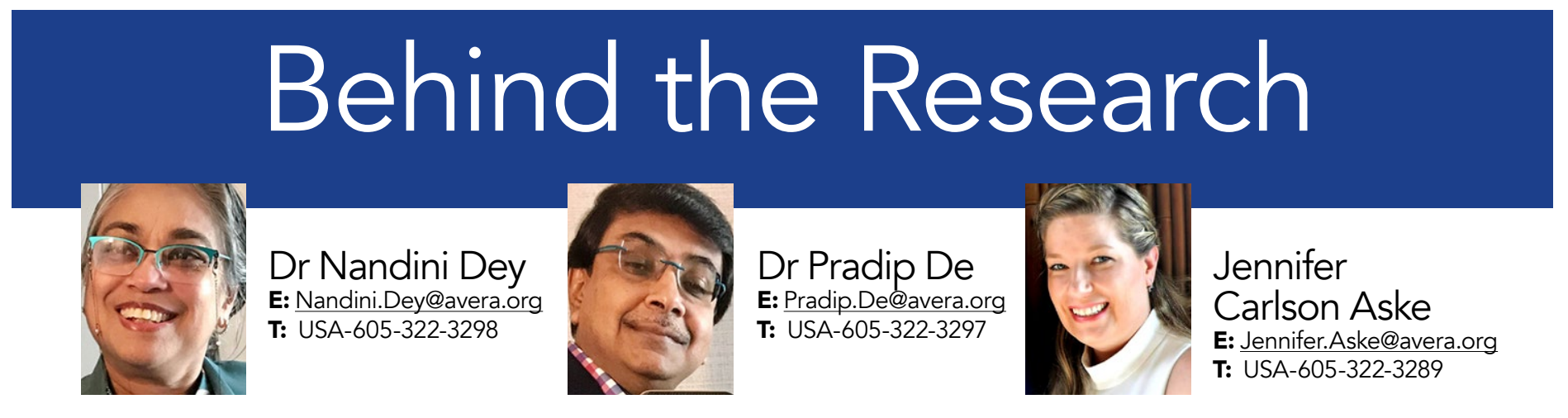

Research Objectives

The Avera Cancer Institute team study anti-cancer drugs and their mode of action, in the contex
including cell death by apoptosis.

\section{Detail}

Dr Nandini Dey

Translational Oncology Laboratory, Avera Cancer Institute, 1000 E 23rd Street, Sioux Fall, SD, 57105, USA rd Floor of Prairie Center, Suite \# 3611; Room \# 3610

Departmental of Internal Medicine, SSOM, University of South Dakota, Sioux Falls, SD 57105, USA

\section{Dr Pradip De}

gy Laboratory, Avera Cancer Institute, 1000 E 23rd Street, Sioux Falls, SD, 57105, USA 3rd Floor of Prairie Center, Suite \# 3611; Room \# 3609 Departmental of Internal Medicine, SSOM, University of South Dakota, Sioux Falls, SD 57105, USA

Consultant, Viviphi, Greenwood Village, CO 80112, USA

Jennifer Carlson Aske

Astaboratory, Genomic Oncology Institute, Plaza 2, 1301 South Cliff Avenue, Plaza 2, Suite 703,

Bio

Dr Nandini Dey, M.S., PhD is a Senior Scientist, \& Director of Translational Oncology Laboratory, Avera Cancer Institute, with a special interest in cell phenotypes. Dr Dey is a member Whe

Dr Pradip De, M.S., PhD is a Senior Scientist of the laboratory with 25 years' experience in signal transduction biology. Together, Dey \& De have over 50 years' experience in translational research and have authored or co-authored over 100 publications.

Mrs Jennifer Carlson Aske, M.S. is the Research Laboratory Supervisor with 10 years of research experience.

Funding

\section{References} De, P., Carlson, J., Leyland-Jones, B., Williams, C. and Dey, N.
(2018). Triplef fluorescence staining to evaluate mechanism-based apoptosis following chemotherapeutic and targeted anti-cancer
drugs in live tumour cells. Sci Rep, 8, 13192-13202. Available drugs in live tumour cells. Sci Rep, 8, 13192-13202. Available
at: https://www.nchbinlm.nih.gov/pmc/articles/PMC6123436/ at: https://www.nchi.nlm
[Accessed 18.03.2019]

Ulukaya, E., Acilan, C. and Yilmaz, Y. (2011). Apoptosis: why and how does it occur in biology? Cell Biochem and Function, 29 (6), 468-480. Avaliable at: https://onlinelibrary.wiley.com/doil abs/10.1002/cbf.1774 [Accessed 18.03.2019]

\section{Personal Response}

Why would some cells show red and blue staining in the "ive and dead cell assay'?

II The live and dead cell assay' was carried out to show live cells as green and dead cells as red. There is no blue colour in the assay. During the 'triple fluorescence staining' under treatment conditions because, during the initial stage of apoptosis, mitochondria are still functional (blue) while their cell membranes have just started to show apoptotic features (red). These are really rare events and dependent on the drug used to induce apoptosis. In culture conditions,
all cet in the same stage of apoptosis, and all cells were not synchronized for their stages of cell cycle.

\section{What do you see for the future of this research?}

II Considering the laboratory friendly nature of the 'triple-fluorescence staining' in live cells, we expect that
this method will become a commonly used laboratory tool to study drug effects in cancer research.

Although currently the protocol is validated and used in translational oncology, we think that his procedure will be For example the phenomenon of apoptosis is a citicat event in the field of developmental biology, regenerative medicine, and stem cells research. We envision that studies in the field of Parkinson's disease, Alzheimer's disease, myocardial ischaemia, myocardial infarction, and traumaprotecol effectively. 